\title{
Effect of System Pressure on the Surface Microstructure of Spherical Porous Basic Magnesium Carbonate
}

\author{
Yu Wei ${ }^{1}$, Hong Lei ${ }^{1}$,Weikang Peng ${ }^{1}$ and Zhiliang Huang ${ }^{1, \text { a }}$ \\ ${ }^{1}$ School of Materials Science and Engineering, Wuhan Institute of Technology, Wuhan 430074, People's Republic of \\ China
}

\begin{abstract}
Mg}_{5}\left(\mathrm{CO}_{3}\right)_{4}(\mathrm{OH})_{2} 4 \mathrm{H}_{2} \mathrm{O}\right)$ has been prepared by using ammonium bicarbonate and magnesium chloride hexahydrate as raw materials. A novel $\mathrm{CO}_{2}$ bubble template was constructed to explain the formation of pore structure of porous $\mathrm{Mg}_{5}\left(\mathrm{CO}_{3}\right)_{4}(\mathrm{OH})_{2} 4 \mathrm{H}_{2} \mathrm{O}$. System pressure was found to be important in controlling the final pore size of spherical porous $\mathrm{Mg}_{5}\left(\mathrm{CO}_{3}\right)_{4}(\mathrm{OH})_{2} 4 \mathrm{H}_{2} \mathrm{O}$. By the investigation of the particles from pressure-dependent experiments, scanning electron microscopy observations, X-ray diffraction, and Fourier transform infrared spectra were used to follow the reactions and identify the products. The results show that the pore size of spherical porous $\mathrm{Mg}_{5}\left(\mathrm{CO}_{3}\right)_{4}(\mathrm{OH})_{2} 4 \mathrm{H}_{2} \mathrm{O}$ gradually decreased via increasing system pressure.
\end{abstract}

\section{Introduction}

For magnesium resource utilization, the new development direction is that the abundant magnesium chloride is used to synthesize the functional magnesium carbonate with excellent performance. Basic magnesium carbonate (BMC) is a kind of important magnesium inorganic material. With good fluidity, filling and dispersion [1], BMC has played an more and more important part in many industries and fields, such as pharmaceuticals, rubber industry, insulation materials, dental preparations, senior glass, food industry and ingredients of various kinds of chemical products [2].At present, many studies about preparation of BMC have been reported and most crystal microstructure are spherical[3], rod-like [4] and microtubes [5]. Because of the larger specific surface area and higher decomposition temperature, spherical structure attract extensive attention, many methods were used to synthesis BMC. For example, Zaki et al have synthesized spherical BMC by using $\mathrm{Cr}$ solution as promoter [6]. However, the study on the surface microstructure of spherical porous basic magnesium carbonate is rare. It is important to control the surface microstructure with simple condition.

In this paper, the spherical porous BMC was synthesized without using surfactants. It is supposed the assembling of spherical porous $\mathrm{BMC}$ with $\mathrm{CO}_{2}$ bubble template according to phase transformation. We try to use several equation to describe relationship between pore size and pressure. Further more, Effect of system pressure on the surface microstructure of spherical porous basic magnesium

\footnotetext{
${ }^{a}$ Corresponding author: hz16455@126.com
} 
carbonate was studied.

\section{Experimental}

\subsection{Materials and Synthesis}

Magnesium chloride hexahydrate and ammonium bicarbonate were used as analytical grade in a typical experiment. Then, aqueous solution were transferred into special autoclave. The pressure of the system can be accurately adjust at any time, and the system temperature remains steady at $373 \mathrm{k}$. After reaction finished, the special autoclave was taken out to age for $5 \mathrm{~h}$, and the mixture was repeatedly washed by distilled water and ethanol. After that, vacuum oven was used to dry mixture. Finally, the solid products were obtained at different system pressure as follows: $S_{1}, 4$ atm; $S_{2}, 6$ atm; $\mathrm{S}_{3}, 8 \mathrm{~atm} ; \mathrm{S}_{4}, 10 \mathrm{~atm}$.

\subsection{Characterization}

Fourier transform infrared and powder X-ray diffraction were employed to analyze the chemical composition of BMC. A scanning electron microscope was used to observe The microstructure and the surface changes of spherical BMC.

\section{Results and discussion}

Spectra for the obtained products at different system pressure are shown in Fig.1, the spectrum of the $\mathrm{S}_{1}$ synthesized at $4 \mathrm{~atm}$ shows typical bands at 3648.76, 3514.54, 3448.70, 797.74 and $593.77 \mathrm{~cm}^{-1}$ which are due to structural hydroxyl $(\mathrm{O}-\mathrm{H})$, with those at 1483.55, 1421.56, 1120.24, 885.31, 852.99 and $713.11 \mathrm{~cm}^{-1}$ are due to carbonate anion $\left(\mathrm{CO}_{3}{ }^{2-}\right)$. Apparently, spectra of $\mathrm{S}_{2}, \mathrm{~S}_{3}, \mathrm{~S}_{4}$ are similar to $\mathrm{S}_{1}$, XRD patterns of $S_{1}, S_{2}, S_{3}$ and $S_{4}$ were shown in Fig. 2, According to the standard JCPDS card NO. 25-0513, it is obviously that all those samples are considered to be BMC with high purity crystalline. In addition, An interesting phenomenon was found from the 4 patterns was that diffraction peaks become more acute with the increase of pressure. Thus, from the analysis of the XRD and FT-IR, all of the products are BMC.

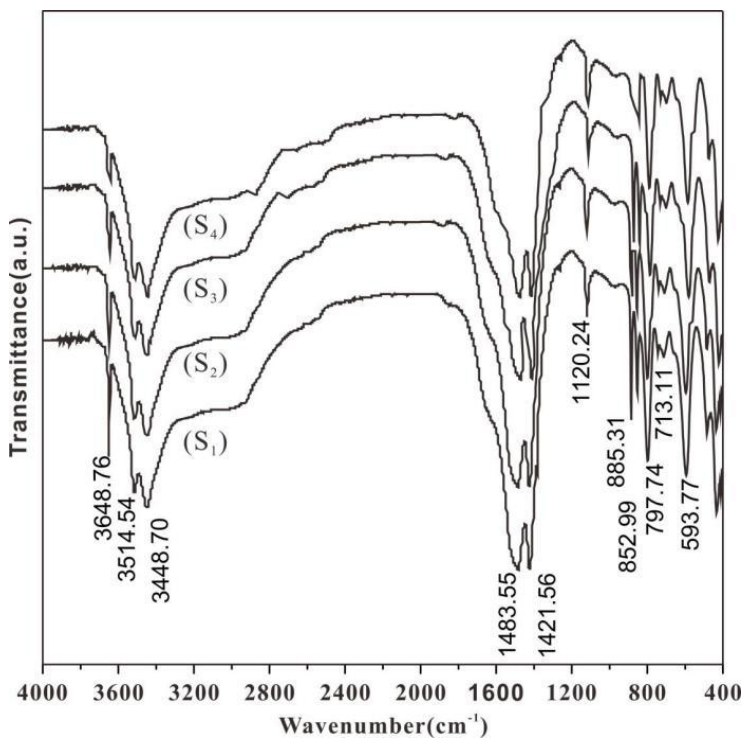

Figure 1. Infrared spectra of solid products synthesized at different system pressures $\left(\mathrm{S}_{1}: 4 \mathrm{~atm} ; \mathrm{S}_{2:} 6 \mathrm{~atm} ; \mathrm{S}_{3}: 8\right.$ atm; $\left.\mathrm{S}_{4}: 10 \mathrm{~atm}\right)$ 


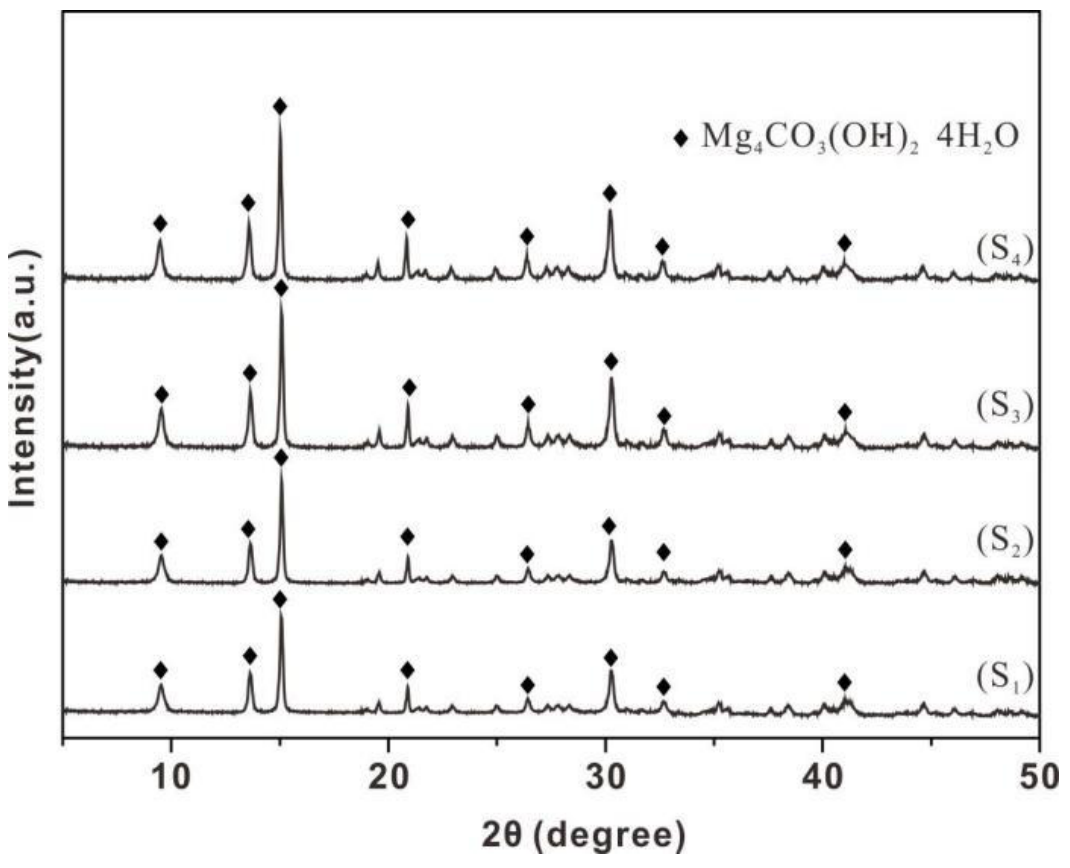

Figure 2. XRD pattern of samples synthesized at different system pressures $\left(\mathrm{S}_{1}: 4 \mathrm{~atm} ; \mathrm{S}_{2}: 6 \mathrm{~atm} ; \mathrm{S}_{3}: 8 \mathrm{~atm} ; \mathrm{S}_{4}\right.$ : $10 \mathrm{~atm})$

SEM images of $S_{1}$ is shown in Fig. 3, the surface of BMC is assembled by the flakes, most of which interconnect and form an porous sphere. Cheng et al have discovered that gas bubbles can be seen as a type of template for crystal growth, In this paper, $\mathrm{CO}_{2}$ bubbles is considered to be a temple to form such porous sphere of BMC. Growth process of $\mathrm{Mg}_{5}\left(\mathrm{CO}_{3}\right)_{4}(\mathrm{OH})_{2} \cdot 4 \mathrm{H}_{2} \mathrm{O}$ is shown Figure 4. The existence and aggregation of many bubbles lead to form a foam system. In this system. the plateaus emerge with highest energy density is in the center of at least three $\mathrm{CO}_{2}$ bubbles where $\mathrm{BMC}$ begin to nucleate steadily. After that, BMC grow up via the the passage between the $\mathrm{CO}_{2}$ bubbles by self-assembling. Lastly, when reaction is over, $\mathrm{CO}_{2}$ bubbles was washed and spherical porous basic magnesium carbonate was obtained.

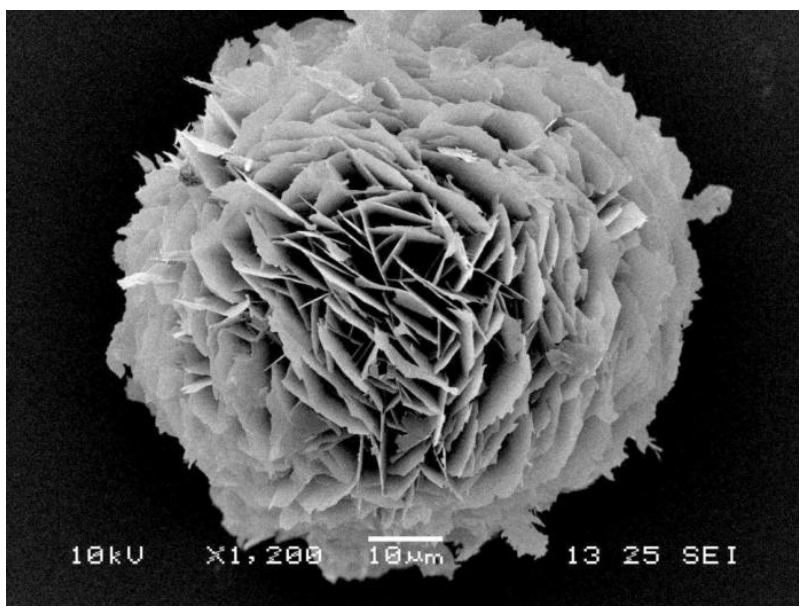

Figure 3. SEM images of $S_{1}$. 

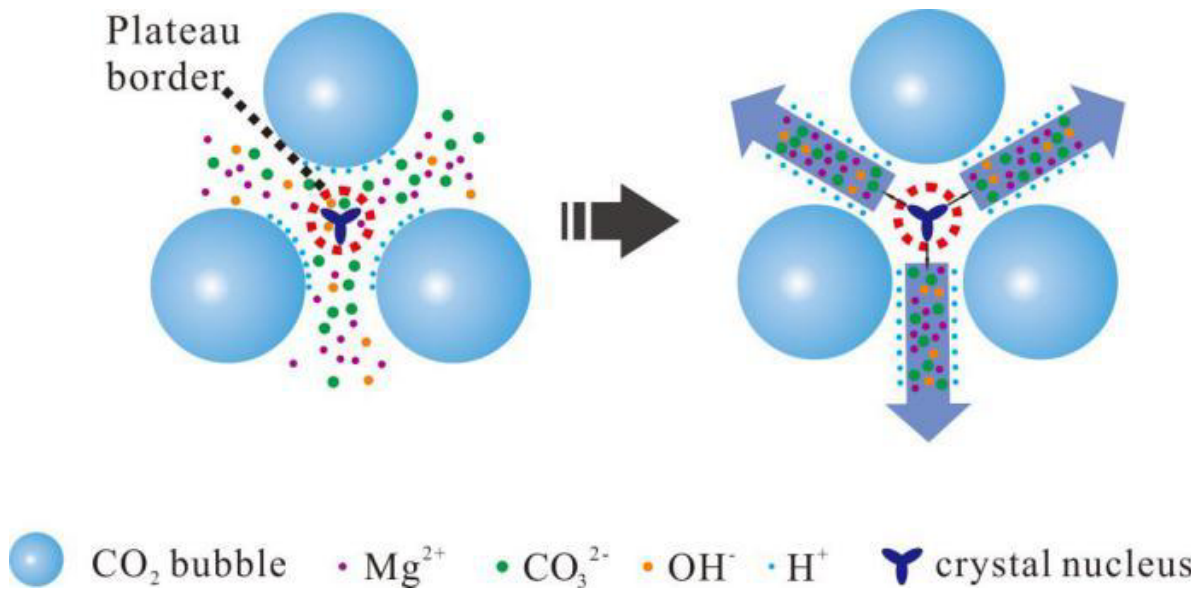

Figure 4. Illustration of the growth process of the flakes of BMC.

The effect of the system pressures on the Pore Size of the spherical porous BMC was studied. Fig.5 shows SEM images of the BMC obtained under different system pressures. BMC microspheres were found different under different given pressures. It is obvious that the arrangement of lamellae is denser with the system pressures increasing. Due to the increased system, volume of $\mathrm{CO}_{2}$ bubbles will become smaller. Then, the channel between the $\mathrm{CO}_{2}$ bubbles become narrower and the distance between the adjacent channels decrease, which results in smaller lamellae and denser arrangement of lamellae. This phenomena indicated that with increasing system pressure, the pore sizes of BMC microspheres decreased.
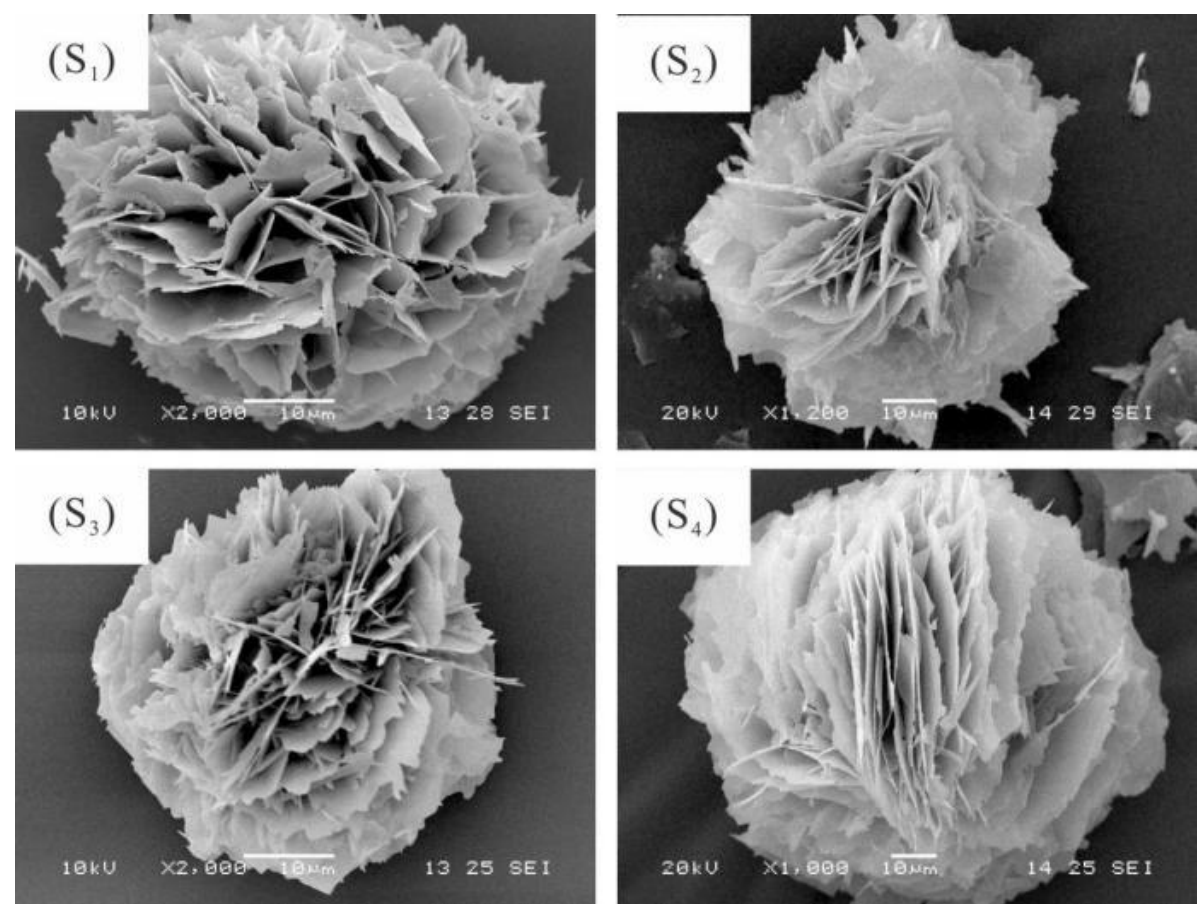

Figure 5. SEM images of spherical porous BMC synthesized at different system pressures $\left(\mathrm{S}_{1}: 4 \mathrm{~atm} ; \mathrm{S}_{2:} 6 \mathrm{~atm}\right.$; $\left.\mathrm{S}_{3}: 8 \mathrm{~atm} ; \mathrm{S}_{4}: 10 \mathrm{~atm}\right)$

According to the viewpoint of thermodynamics, $\mathrm{CO}_{2}$ bubble is constant in solution, which can be described by Laplace, Kelvin equation and Clapeyron equations as follows [7] : 


$$
\begin{gathered}
\Delta P=P_{\text {in }}-P=\frac{2 \delta}{r} \\
P_{\text {in }}=\frac{n R T}{\frac{3}{4} r^{3}}+P_{r}^{*} \\
\ln \frac{P_{r}^{*}}{P^{*}}=-\frac{2 d M}{\rho R T r}
\end{gathered}
$$

According to the three equations 1, 2 and 3 above, combination equation can be obtained :

$$
P=\frac{n R T}{\frac{4}{3} r^{3}}+P^{*} \exp \left(-\frac{2 \delta M}{\rho R T r}\right)-\frac{2 \delta}{r}
$$

The final Equation 4 reveals the increasing pressure $P$ has a major impact on the radius of $\mathrm{CO}_{2}$ bubble, which results in smaller pore size of $\mathrm{Mg}_{5}\left(\mathrm{CO}_{3}\right)_{4}(\mathrm{OH})_{2} 4 \mathrm{H}_{2} \mathrm{O}$ with increasing pressure mentioned above. Thus, it proves that the formation of pore structure is based on $\mathrm{CO}_{2}$ bubbles as template.

\section{Conclusions}

(1) spherical porous $\mathrm{Mg}_{5}\left(\mathrm{CO}_{3}\right)_{4}(\mathrm{OH})_{2} 4 \mathrm{H}_{2} \mathrm{O}$ have been prepared by using ammonium bicarbonate and magnesium chloride hexahydrate as raw materials.

(2) System pressure was important in controlling the final pore size of spherical porous BMC. In addition, with increasing pressure, the pore sizes of BMC microspheres decreased.

(3) A novel $\mathrm{CO}_{2}$ bubble template was constructed to explain the formation of pore structure of porous $\mathrm{BMC}$. And the theoretic equation is described by Laplace, Kelvin equation and Clapeyron equations.

\section{References}

1. D. Bhattacharjya, T. Selvamani, I. Mukhopadhyay, J. Therm. Anal. Calorim. 107, 439-445 (2011)

2. H. Pang, P. Tian, J. Wang, X. Wang, G. Ning, Y. Lin, Mater. Lett. 131, 206-209 (2014)

3. X. Ma, Q. Zhang, Z. Luo, X. Lin, G. Wu, Mater. Design. 89, 71-77 (2016)

4. C. Yan, D. Xue, J. Phy. Chem. B. 109, 12358-12361 (2005)

5. Y.P. Jiang, T.J. Peng, H.J. Sun, Adv. Mater. Res. 178, 230-235 (2010)

6. M.I. Zaki, S.A.A. Mansour, J.Mater. Sci. Let. 13 505-507 (1994)

7. X.H. Zhang, A. Khan, W.A. Ducker, Phys. Rev. Lett. 98, 136101 (2007) 